\title{
ANISOTROPIC LATTICE MISFIT RELAXATION IN AlGaAs SEMI-BULK LAYERS GROWN ON GaAs SUBSTRATES BY LIQUID PHASE ELECTROEPITAXY*
}

\author{
Z.R. ŻyTkiewicz, J. Domagala, J. Bąk-Misiuk, D. DobosZ \\ Institute of Physics, Polish Academy of Sciences \\ Al. Lotników 32/46, 02-668 Warsaw, Poland
}

AND M. LESZCZYŃSKI

High Pressure Research Center, Polish Academy of Sciences Sokołowska 29/37, 01-142 Warsaw, Poland.

Experimental evidence for unidirectional microcracking in semi-bulk AlGaAs layers grown on (001) GaAs substrates is presented. The asymmetrical microcracking leads to anisotropic lattice misfit relaxation in the $\mathrm{AlGaAs} / \mathrm{GaAs}$ structure and is explained in terms of higher mobility of $[-110]$-oriented $\alpha$-type dislocations than that of $\beta$-type dislocations oriented in [110] direction.

PACS numbers: 68.55.Ln, 68.60.Bs

\section{Introduction}

The lattice mismatch across an interface between the epitaxial film and its substrate can be accommodated by a uniform elastic strain until the critical film thickness is reached. Thereafter, it is energetically favourable for the misfit to be shared between a grid of dislocations at the interface and residual strain in the layer. Considerable research activity has been developed to study the mechanisms by which the misfit strain relaxation occurs. Despite the progress made, our understanding of these problems is still insufficient.

In this report, we present some novel observations on the misfit strain relaxation in AlGaAs layers grown on GaAs substrates. The lattice misfit relaxation in this system has been studied very intensively. This was because of a wide application of the AlGaAs/GaAs heterostructures in optoelectronics. On the other hand, this subject is also very interesting from the scientific point of view, because

*This work is supported in part by the Committee for Scientific Research grant No. 8 T11B 03010. 
AlGaAs/GaAs is a unique system in which the perfect lattice match of the layer and the substrate occurs at the growth temperature [1]. Therefore, the AlGaAs layers are grown on GaAs substrates without any misfit whereas, due to their different thermal expansion coefficients, the misfit strain and the misfit defects develop only on cooling. Up to now, due to the technological limitations, only thin AlGaAs films could be analysed. In this work, we have examined semi-bulk samples in which quite new effects could be expected. Moreover, we were able to study the same samples before and after GaAs substrate removal making our analysis more complete.

\section{Samples and experimental techniques}

The AlGaAs layers were grown on (001) GaAs substrates by liquid phase electroepitaxy (LPEE) technique at the temperature of $900^{\circ} \mathrm{C}$. The details of the growth procedure can be found elsewhere [2]. However, for the analysis presented here it is important to mention that the unique feature of LPEE grown multicomponent layers is their high compositional uniformity. The layers were Si doped $\left(n \approx 10^{17} \mathrm{~cm}^{-3}\right)$ and were $800 \mu \mathrm{m}$ thick. Before the X-ray studies they were lapped to the thickness of $600 \mu \mathrm{m}$. The microstructure of the samples was examined using high-resolution X-ray diffractometry. The application of a four-reflection Bartels monochromator and a two-reflection analyser ensured the measurements of lattice parameters with ppm accuracy. Moreover, our experimental setup allowed us to examine the samples by the reciprocal lattice mapping technique. The X-ray topographs were obtained using a double-crystal diffractometer set in a non-dispersive mode. The samples were etched in molten KOII to determine the crystallographic directions in the growth plane [3].

\section{Results}

The aluminium content in the layer $x=0.41$ was determined by measuring the lattice constant of AlGaAs. The value obtained was very close to $x=0.39$ measured by scanning electron microprobe. No composition gradient was detected in the [001] growth direction. The reciprocal space mapping for the symmetrical (004) Bragg reflection showed absence of mosaic structure in the samples. Table presents the values of the FWHM of the (004) rocking curves measured for the AlGaAs layers. As can be seen, the rocking curves were broader with respect to perfect AlGaAs layers grown by MBE, for which the FWHM of $15 \operatorname{arcsec}$ is

TABLE

Curvature radii and the values of FWHM of (004) rocking curves of AlGaAs.

\begin{tabular}{c|c|c|c|c}
\hline \hline \multirow{2}{*}{$\begin{array}{c}\text { Direction in } \\
\text { (001) plane }\end{array}$} & \multicolumn{2}{|c|}{ AlGaAs layer on GaAs substrate } & \multicolumn{2}{c}{ Free-standing AlGaAs layer } \\
\cline { 2 - 5 } & $\begin{array}{c}\text { FWHM } \\
\text { [arcsec] }\end{array}$ & $\begin{array}{c}\text { curvature radius } \\
{[\mathrm{m}]}\end{array}$ & $\begin{array}{c}\text { FWHM } \\
\text { [arcsec] }\end{array}$ & $\begin{array}{c}\text { curvature radius } \\
{[\mathrm{m}]}\end{array}$ \\
\hline$[110]$ & 48 & $R_{1}=7$ & 20 & $R_{1}=\infty$ \\
{$[-110]$} & 72 & $R_{2}=2$ & 38 & $R_{2}=6$
\end{tabular}




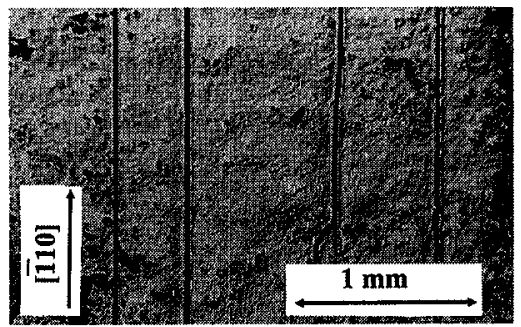

Fig. 1. Optical photomicrograph showing the [-110]-oriented microcracks on the bottom face of LPEE grown AlGaAs layer. The GaAs substrate was removed by chemical etching.

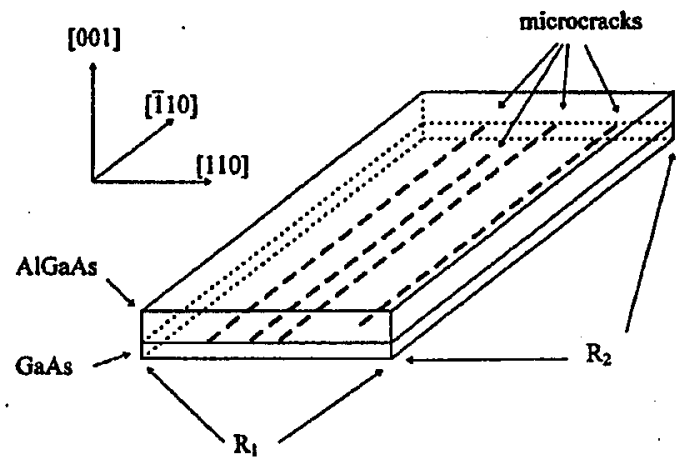

Fig. 2. Schematic drawing of AlGaAs/GaAs structure. The position and orientation of microcracks together with the definition of curvature radii $R_{1}$ and $R_{2}$ in two $\langle 110\rangle$ directions are shown.

usually observed under our experimental conditions. This indicated some strain and/or defects present in LPEE grown AlGaAs layers. As a matter of fact, X-ray topography of the layers revealed a network of misfit dislocations lying in two orthogonal $\langle 110\rangle$ directions. When the substrate was removed by chemical etching the rocking curves became narrower with the value of FWHM close to our experimental limit. Moreover, the curvature radii of the layers increased significantly. Thus, the broadening of the rocking curves as well as pronounced bending of the layers showed that AlGaAs layers were placed in compression by the GaAs substrate. Finally, the $[-110]$-oriented microcracks lying close to the interface in the AlGaAs layer (see Fig. 1) were detected by selective etching and optical microscopy. These microcracks are schematically shown in Fig. 2 in order to correlate their position and orientation with the bending anisotropy of the samples.

\section{Discussion}

In order to interpret our results let us assume that due to the perfect lattice match to the GaAs substrate at the growth temperature, no lattice relaxation occurs during growth of the AlGaAs layer. In fact, some localised strains caused by terracing, meniscus lines or roughness of the substrate surface (typical defects in 
LPE or LPEE grown layers [4]) may relax even at the growth temperature. Nevertheless, we believe that this has a minor influence on the relaxation anisotropy observed. During the cooling after the growth is terminated, the AlGaAs layer experiences the compressive stress which appears due to a different thermal expansion of $\mathrm{AlGaAs}$ and GaAs. Although the resulting lattice misfit is low $(\approx 0.13 \%$ at room temperature [1]), our layers are much thicker than the critical thickness, which for the AlGaAs/GaAs system is estimated to be in the range of $10 \mu \mathrm{m} \mathrm{[1]}$. Therefore, as visible in X-ray topographs, an array of the $60^{\circ}$ misfit dislocations oriented in two orthogonal $\langle 110\rangle$ directions appears at the interface. Because of the high dislocation density in the substrate $\left(5 \times 10^{4} \mathrm{~cm}^{-2}\right)$ misfit dislocations develop most probably via bending in the interface plane of the dislocations threading from the substrate, i.e. by the mechanisms proposed by Matthews et al. [5]. As long as the cooling proceeds, but the temperature is still higher than the so-called freezing temperature $T_{\mathrm{f}}$, further relaxation of the lattice misfit takes place by increasing the length of misfit segment of dislocations and by reactions between the dislocations leading to their multiplication and/or annihilations. Yamamoto and Yamaguchi [6] have determined the value of $T_{\mathrm{f}}$ to be $450 \pm 90^{\circ} \mathrm{C}$ and $250 \pm 100^{\circ} \mathrm{C}$ for the GaAs/Si and InP/Si systems, respectively. Finally, when the temperature is lower than $T_{\mathrm{f}}$, the mobility of the dislocations becomes very low and all the further residual strain caused by cooling from $T_{\mathrm{f}}$ to the room temperature can be accommodated only elastically. This residual thermal strain can be withdrawn from the structure by etching off of the substrate. This is the reason why we have measured the significantly lower bending as well as the lower values of the rocking curves FWHM for the free standing AlGaAs layer as compared to the complete AlGaAs/GaAs structure.

Let us discuss now the mechanism by which microcracks are generated at the AlGaAs/GaAs interface. Cottrell [7] has suggested a fracture mechanism whereby dislocations on intersecting slip planes combine to form sessile dislocations whose missing half planes may be viewed as microcracks. Repeated slip of this type can cause the microcracks to grow. The AlGaAs layers are under compressive strain which would tend to keep the microcracks from opening up and thereby inhibit complete fracture. The dislocations observed in our X-ray topographs were oriented in [-110] and [110] crystallographic directions. They are known as $\alpha$ and $\beta$ dislocations lying on $\{111\}_{\mathrm{As}}$ and $\{111\}_{\mathrm{Ga}}$ planes, respectively [8]. Since the mobilities of these two types of dislocations are not expected to be equal, it follows that the one set will move faster and at lower stress levels than the other, and thus give rise to fracture which appears only along one direction despite the fact that the misfit strain is applied equally along the two directions. In fact it is known that in $n$-type GaAs the $\alpha$-type dislocations are much more mobile than those of $\beta$-type [9]. As follows from Fig. 1 the same must hold true for our AlGaAs layers as an unidirectional set of $[-110]$ oriented microcracks is observed. It is obvious that the microcracks formation is an additional mechanism by which the lattice misfit can be relaxed. This explains why the value of the curvature radius $R_{1}$ measured in the [110] direction is much larger than that $R_{2}$ in the [-110] direction (see Table). Finally, it is worth mentioning that the asymmetric cracking has been already observed in $\mathrm{GaAsP} / \mathrm{GaAs}$ and $\mathrm{InGaP} / \mathrm{GaAs}$ systems [8]. As 
a rule, cracking appeared more often in samples under tensile strain (as in the $\mathrm{GaAsP} / \mathrm{GaAs}$ and InGaP/GaAs systems). Beside our results on AlGaAs/GaAs structures, the cracking of the compressive stressed InGaP/GaAs samples [8] is, to the best knowledge of the authors, the only published observation of fracture in III-V heterostructures under the compressive strain.

\section{Summary}

We have proved that the semi-bulk AlGaAs layers grown on GaAs substrates by LPEE technique are of the high crystallographic perfection. Beside the well known mechanism of the lattice misfit relaxation by the formation of the two-directional grid of misfit dislocations an additional mechanism of lattice relaxation by the formation of unidirectional set of microcracks in the plane of the AlGaAs/GaAs interface is operative during cooling of the structure between the growth and freezing temperatures. Our results indicate that in $n$-type AlGaAs alloy the mobility of $\alpha$-type dislocations is larger than that of $\beta$-type which leads to the easier fracture in the $[-110]$ direction and explains the observed anisotropy of lattice misfit relaxation. We show that despite the compressive nature of the strain in the AlGaAs epitaxial layer the microcracking may occur, which is the very rare case among the wide variety of III- $V$ heterostructures reported in the literature.

\section{References}

[1] M.C. Roland, D.A. Smith, J. Cryst. Growth 38, 143 (1977).

[2] Z.R. Żytkiewicz, J. Cryst. Growth 146, 283 (1995).

[3] G. Nozle, V. Geist, G. Wagner, P. Paufler, K. Jurkschat, Z. Kristallogr. 193, 111 (1990).

[4] W.T. Stacy, J.J. Daniele, J. Cryst. Growth 44, 23 (1978).

[5] J.W. Matthews, S. Mader, T.B. Light, J. Appl. Phys. 41, 3800 (1970).

[6] A. Yamamoto, M. Yamaguchi, Mater. Res. Soc. Symp. Proc. 116, 285 (1988).

[7] A.H. Cottrell, Trans. AIME 212, 192 (1958).

[8] G.H. Olsen, M.S. Abrahams, T.J. Zamerowski, J. Electrochem. Soc. 121, 1650 (1974).

[9] I. Yonenaga, K. Sumino, J. Appl. Phys. 65, 85 (1989). 